\title{
Estimation of Hybrid Vigour for Fruit Yield and Quality Traits of Okra [Abelmoschus esculentus (L.) Moench] Through Line x Tester Analysis Carried Over Environments
}

\author{
Sanket J. More $^{1 *}$, K.N. Chaudhari ${ }^{2}$, G.B. Vaidya ${ }^{3}$ and S.L. Chawla ${ }^{2}$ \\ ${ }^{1}$ ICAR-Central Tuber Crops Research Institute, Sreekariyam PO, Thiruvananthapuram, \\ Kerala 695 017, India \\ ${ }^{2}$ ASPEE College of Horticulture and Forestry, Navsari Agricultural University, \\ Navsari 396 450, India \\ ${ }^{3}$ ANKUR seed co. PVT. LTD., Nagpur, India \\ *Corresponding author
}

\section{A B S T R A C T}

Heterosis is one of the greatest practical achievements of plant breeding and has been extensively used in crop improvement in okra. The production of hybrid cultivars with higher fruit yield and quality characters has become increasingly important in recent years due to continuous demand. In the present study, 14 genetically diverse okra parents were crossed in Line $\mathrm{x}$ Tester fashion to explore magnitude of heterosis. Pooled analysis of 3

Keywords

Environment, Hybrid vigour, Okra, Quality traits and Yield.

Article Info

Accepted:

29 June 2017

Available Online:

10 July 2017 environments data showed that the maximum extent of significant standard heterosis for fruit yield in desired directions was recorded in hybrids from the crosses VIO $47672 \mathrm{x}$ GJO - $3(33.33 \%)$, IC - 045796 x GJO - 3 (22.83\%), IC - 111493 x GJO - 3 (22.27\%). A pursue of per se performance revealed that the hybrid from the cross VIO $47672 \times$ GJO - $3\left(\mathrm{E}_{1}: 9.66 \mathrm{t} / \mathrm{ha}, \mathrm{E}_{2}: 15.58 \mathrm{t} / \mathrm{ha}, \mathrm{E}_{3}: 5.65 \mathrm{t} / \mathrm{ha}\right.$ and pooled: $\left.10.30 \mathrm{t} / \mathrm{ha}\right)$ was found to be performing consistent over environments and in pooled as well. Performance of these hybrids should be further tested for commercial cultivation. Hybrids, VIO 44244 x Arka Anamika (-8.80 \%), VIO 44244 x GJO - 3 (-9.48 \%) and AOL-09 -17 x GJO- 3 (19.88 $\%)$ exhibited significant standard heterosis in desired direction for stalk length, fiber content and shelf life, respectively. Stalk colour is an attractive trait for visualization of diverse okra genotypes in which dark green stalk colour is most preferable for edible purpose. Hybrids from the crosses viz, IC - 045796 x GJO - 3, IC - 052273 x GAO - 5, IC - 052273 x Varsha Uphar, VIO 47672 x GJO - 3 and EC - 305623 x GAO - 5 exhibited dark green stalk colour. On the basis of per se performance and heterosis analysis for fruit yield per plant hybrid VIO 47672 x GJO - 3 was the best followed by IC - 111493 x GJO - 3, IC - 045796 x GJO - 3 and performance of these hybrids should be further tested for commercial cultivation.

\section{Introduction}

Okra (Abelmoschus esculentus (L.) Moench) belongs to family Malvaceae, is an important fruit vegetable grown throughout the tropics and warmer parts of the temperate zone
(Kumar et al., 2016). Tender okra fruits are used as vegetable in India, Brazil, West Africa and many other countries. Because of high nutritive value and prolonged shelf life, 
okra captured prominent position among the export oriented vegetable crops and thus act as one of the valuable foreign exchange earner crop (Patel, 2014). Okra is a polyploid, with most observed chromosome number from $2 n=56$ to 199 and an often cross pollinated crop. Highly variable cross pollination rate from 0 to $69 \%$ has been recorded for $A$. esculentus (Anonymous, 2011; Charrier, 1984). India is a major okra producing country in the world comprising of $72 \%$ of total area under okra (FAOSTAT, 2013). The area under okra cultivation in India is 504 (000 ha) with production of 5709 (000 MT) and productivity of $11.33 \mathrm{MT} / \mathrm{ha}$, containing 5.03 per cent of the total area under vegetable crops and 3.18 per cent of total vegetable production (Anonymous, 2015). Extensive use of local landraces or traditional varieties and limited availability or improved high yielding varieties resulted in stable productivity of okra in India over the years (Reddy et al., 2012). These facts warrant development of high yielding varieties for commercial cultivation. Since, Vijayaraghavan and Warrier (1946) reported heterosis in okra for the first time, hybrid vigour studies have been explored extensively. Several research workers reported the presence of heterosis in desired quantities for fruit yield and its various components (Kishor et al., 2013; Obiadalla et al., 2013; Kumar et al., 2014; Akotkar et al., 2014; Badiger et al., 2014; Patel, 2014; Katagi et al., 2015). The productivity of okra should be increased through hybridization and recombination.

\section{Materials and Methods}

The experiment was conducted between September, 2013 and February, 2015 (including crossing programme during September to December, 2013). The experiment consisting 55 genotypes including 14 parents their 40 hybrids and one standard check, was laid out in a Randomized Block Design (RBD) with three replications over three seasons (Table 1) at the Regional Horticultural Research Station, ASPEE College of Horticulture and Forestry, Navsari Agricultural University, Navsari, situated at coastal region of South Gujarat. Geographically, it is situated at $20^{\circ}-37^{\prime} \mathrm{N}$ latitude and $72^{\circ}-54^{\prime} \mathrm{E}$ longitude with an altitude of 11.98 meters above the Mean Sea Level. All recommended horticultural practices along with plant protection measures were followed uniformly and timely.

Fourteen diverse parents (Table 2) based on their divergence values were crossed to produce forty $F_{1}$ hybrids. Selfed truthful seeds treated with Thiram $4 \mathrm{~g} / \mathrm{kg}$ of seeds of 14 diverse genotypes were sown in line $\mathrm{x}$ tester fashion in a single-row plot of ten plants spaced at $60 \times 45 \mathrm{~cm}$ in vertisols of Navsari region.

Observations on days to first flowering, internodal length, plant height, number of branches per plant, number of fruits per plant, fruit yield per plant, fruit length, fruit diameter, fruit weight, stalk length, crude fiber content, shelf life at room temperature and stalk colour were recorded in five plant chosen at random in each genotype and in each replication. The crude fiber content from okra fruits was determined by method as described by Thimmaiah (1999). The data pertaining to various traits under study were analyzed as per procedure suggested by Panse and Sukhatme (1967) and Singh and Chaudhary (1977) using following model. Heterosis was estimated as the per cent increase (+) or decrease (-) of $F_{1}$ hybrid mean over the standard check (standard heterosis). For practical plant breeding, the estimation of heterosis over standard check is more realistic. These values were calculated using means over replications and tested for significance as follows: 
Per cent heterosis over $\quad \mathrm{F} 1-\mathrm{SC}$

Standard check (SC) = -----------------X 100

Where,

F1: Mean performance of F1 hybrid

SC: Mean performance of the standard check

The deviations for heterosis were tested for their significance by the following standard errors.

S.E. of difference for

Standard check $=\left[2 \mathrm{M}_{\mathrm{e}} / \mathrm{r}\right]^{0.5}$

Where,

$\mathrm{Me}=$ Error mean square of individual environment

C.D. $=$ S.E. (d) $\mathrm{x}$ 't' at error d.f. $(\mathrm{P}=$ 0.05 and $\mathrm{P} 0.01$ levels of significance)

\section{Results and Discussion}

\section{Analysis of variance}

Pooled analysis of variance over three environments (Table 3) revealed highly significant differences for all the traits, which indicated that the genotypes studied, had reacted differentially to the environments. Significant differences for all the traits (except internodal length, fruit length and fruit diameter and fruit weight) existed among parents over environments. The variance due to parents was further partitioned into variance due to females, males and females vs. males. The results followed the same trend as it was for parents. The mean squares due to males and females were highly significant for all traits in individual as well as pooled over environments (except days to first flowering, internodal length, number of branches per plant, fruit yield per plant, fruit length and fruit weight in case of males, while, internodal length, fruit length and fruit weight in the case of females).

The interaction of hybrids $\mathrm{x}$ environments were significant for all the traits except fruit length and fruit weight which indicated influence of environment on the expression of various traits.

The average performance of hybrids was different from that of parents in different environments which were evident from the significance of parents' $v / s$ hybrids $\mathrm{x}$ environment interaction for various traits. This is in argument with several researchers (Sharma and Singh, 2012; Kishor et al., 2013; Kumar et al., 2014; Das et al., 2013; Badiger et al., 2014).

\section{Per se performance}

For any okra hybrid to be acceptable for commercial cultivation, it should possess significant superiority in fruit yield over the existing commercial hybrid. In the present study, the performance of 40 hybrids was compared with the hybrid 'Sonakshi' which is popular in South Gujarat region.

A perusal of mean values of fruit yield per plant (Table 4) revealed that in general the performance of parents and their hybrids were better under $\mathrm{E}_{2}$. On pooled basis, the hybrid from the cross VIO 47672 x GJO - 3 was found to be the best in respect to fruit yield per plant. High yielding hybrid, in general, had high per se performance genotype as one of its parent and this hybrid significantly surpassed its parents as well as the check.

The wide range of per se performance of the parents for the traits studied suggest the prevalence of considerable amount of genetic variability which is one of the causes of 
heterosis. Deviation observed in the per se performance of the hybrids compared to that of the parents is also an indication of the manifestation of heterosis in the hybrids.

The above results are in conformity with Badiger et al., (2014). Various environmental parameters viz; rainfall, temperature, humidity and sunshine were recorded over three seasons. In the present study, most of the genotypes had better yield traits in rainy season as compared to summer and winter season.

Seasonal variation was more effective for yield increment, during the rainy season when the rainfall and relative humidity was optimum and temperature and sunshine was low, leading to high heterotic values. Whereas, in summer season, temperature and sunshine was high but rainfall, relative humidity was low and heterosis per cent also decreased for yield.

Heterosis values were the lowest in winter season because of low temperature. This may be due to adverse growing conditions of okra plants in summer and winter as compared to rainy seasons.

\section{Estimation of heterosis for yield and yield components}

The extent of heterosis of top three crosses over standard check "Sonakshi" estimated for 12 traits in individual environments and on pooled basis is presented in tables $5 \mathrm{a}$ and $5 \mathrm{~b}$. It can be seen from the results that the top three heterotic crosses for fruit yield exhibited earliness in flowering. Early flowering hybrids with significant and negative heterosis for days to first flowering were IC 111493 x GJO - 3 (-6.49 \%), VIO 47672 x GJO - $3(-6.37 \%)$ and VIO 47672 x GAO - 5 $(-6.04 \%)$. Plant height and internodal length at fully matured stages is one of the important ideotype in okra for higher yield.

VIO 47672 x GJO - 3 (18.28 \%), IC 111493 x GJO - $3(15.50 \%)$ and IC - 045796 $\mathrm{x}$ GJO $-3(13.79 \%)$ were the top three performer for plant height while in the case of internodal length, top three hybrids were JOL $-08-13$ x GJO $-3(-9.93 \%), J O L-08-13$ $\mathrm{x}$ Varsha Uphar (-9.78\%) and IC - $052273 \mathrm{x}$ Arka Anamika (-8.69\%).

The number of branches per plant and number of fruits per plant are major yield attributing components contributing to higher productivity. Component wise examination of the crosses revealed that most of the high yielding crosses manifested high standard heterosis for number of branches per plant and number of fruits per plant which are major yield components.

VIO 47672 x GJO - $3(20.92 \%$ and 24.15 $\%)$, IC -045796 x GJO - $3(15.18 \%$ and $13.79 \%)$ and IC - 111493 x GJO - 3 (15.16 $\%$ and $15.50 \%$ ) were the top three hybrids with significant and positive standard heterosis for number of fruits and plant height, respectively.

The increase in number of branches and plant height ultimately increased number of fruits which in turn resulted in significant increase in yield (Katagi et al., 2015).

Table.1 Details of seasons taken under study

\begin{tabular}{ll}
\hline Environment & Seasons \\
\hline$E_{1}$ & Summer, 2014 (February to May, 2014) \\
$E_{2}$ & Rainy, 2014 (June to September, 2014) \\
$E_{3}$ & Winter, 2014 - 15 (November, 2014 to February, 2015) \\
\hline
\end{tabular}


Int.J.Curr.Microbiol.App.Sci (2017) 6(7): 4101-4111

Table.2 Parental material used for the study

\begin{tabular}{ll}
\hline Parent & Source/Origin \\
\hline Lines & \\
\hline VIO 44244 & AVRDC, Taiwan \\
IC -111493 & NBPGR, New Delhi, India \\
JOL $-08-13$ & $\begin{array}{l}\text { Junagadh Agricultural University, Junagadh } \\
\text { (Gujarat) }\end{array}$ \\
EC -284327 & NBPGR, New Delhi, India \\
IC -045796 & NBPGR, New Delhi, India \\
IC -052273 & Junagadh Agricultural University, Junagadh \\
& (Gujarat) \\
JOL $-10-18$ & Junagadh Agricultural University, Junagadh \\
AOL $-09-17$ & (Gujarat) \\
VIO 47672 & Anand Agricultural University, Anand (Gujarat) \\
EC -305623 & NBPGR, New Delhi, India \\
\hline Testers & \\
\hline GAO -5 & Anand Agricultural University, Anand (Gujarat) \\
GJO -3 & Junagadh Agricultural University, Junagadh \\
Arka Anamika & (Gujarat) \\
Varsha Uphar & IIHR, Bengaluru, India \\
\hline Commercial Check Sonakshi (F F $_{1}$; Company: Nunhems) \\
\hline
\end{tabular}

Table.4 Per se performance of females, males and hybrids for fruit Yield per plant $(\mathrm{g})$ under individual and pooled over environments

\begin{tabular}{|c|c|c|c|c|c|c|c|c|c|c|c|c|}
\hline \multirow{2}{*}{ Range } & \multicolumn{4}{|c|}{ Females } & \multicolumn{4}{|c|}{ Males } & \multicolumn{4}{|c|}{ Hybrids } \\
\hline & $\mathrm{E}_{1}$ & $\mathrm{E}_{2}$ & $\mathrm{E}_{3}$ & Pooled & $\mathrm{E}_{1}$ & $\mathrm{E}_{2}$ & $E_{3}$ & Pooled & $E_{1}$ & $E_{2}$ & $E_{3}$ & Pooled \\
\hline \multirow[t]{6}{*}{$\overline{\text { Min }}$} & EC- & IC- & JO-08- & EC- & Arka & Varsha & Arka & Varsha & EC- & IC- & IC- & IC- \\
\hline & 305623 & 052273 & 13 & 305623 & Anamika & Uphar & Anamika & Uphar & 305623 & 052273 & 052273 & 045796 \\
\hline & (158.90) & $(229.30)$ & $(86.43)$ & (161.89) & (180.40) & $(190.60)$ & (89.97) & (164.24) & $x$ & $\mathrm{x}$ & $\mathrm{x}$ & $\mathrm{x}$ \\
\hline & & & & & & & & & GAO-5 & GJO-3 & Arka & Varsha \\
\hline & & & & & & & & & (153.75) & ) $(200.36)$ & Anamika & Uphar \\
\hline & & & & & & & & & & & (75.92) & (163.73) \\
\hline \multirow{5}{*}{ Max } & IC- & IC- & JOL-10 & IC- & GAO-5 & GAO-5 & GJO-3 & GAO-5 & VIO & VIO & VIO & VIO \\
\hline & 111493 & 045796 & 18 & 111493 & (213.60) & $(305.77)$ & (95.59) & (204.98) & 47672 & 47672 & 47672 & 47672 \\
\hline & (214.36) & $(306.60)$ & $(107.42$ & $(207.31)$ & & & & & $\mathrm{x}$ & $\mathrm{x}$ & $\mathrm{x}$ & $\mathrm{x}$ \\
\hline & & & & & & & & & GJO-3 & GJO-3 & GJO-3 & GJO-3 \\
\hline & & & & & & & & & (260.90) & & (152.43) & (278.01) \\
\hline
\end{tabular}

Note: $E_{1}, E_{2}$ and $E_{3}$ are different environments viz., Summer - 2014, Rainy - 2014 and Winter - $2014-15$, respectively 
Table.3 Analysis of variance (Mean Squares) for yield and yield components in okra over environments (Pooled basis)

\begin{tabular}{|c|c|c|c|c|c|c|c|c|c|c|c|c|c|}
\hline \multirow{2}{*}{ Source } & \multirow{2}{*}{ d.f. } & \multicolumn{12}{|c|}{ Characters } \\
\hline & & DFF & IL & NOB & $\mathrm{PH}$ & FY & $\mathrm{NF}$ & FL & FD & FW & SL & $\mathrm{CF}$ & SLD \\
\hline Environment & 2 & $1629.4^{* *}$ & $1558.3^{* *}$ & $0.57 * *$ & $5591.32 * *$ & $1366434 * *$ & $5591.3^{* *}$ & $780.77 * *$ & $2.370 * *$ & $560.293 * *$ & $0.516^{* *}$ & $6.689 * *$ & $31.08 * *$ \\
\hline Replication/Environment & 4 & 1.55 & 0.07 & 0.07 & 1.85 & 78.269 & 1.858 & 1.117 & 0.012 & 0.176 & 0.035 & 0.059 & 0.034 \\
\hline Genotype & 53 & $26.63 * *$ & $4.34 * *$ & $0.27 * *$ & $21.56^{* *}$ & $6075.58 * *$ & $21.560 * *$ & $2.122 * *$ & $0.064 * *$ & $1.614 * *$ & $0.075 * *$ & $0.867 * *$ & $1.61 * *$ \\
\hline Parent & 13 & $19.59 * *$ & 0.33 & $0.09 * *$ & $12.17 * *$ & $2842.48 * *$ & $12.177 * *$ & 0.676 & 0.015 & 0.421 & $0.055^{*}$ & $0.399 * *$ & $0.68 * *$ \\
\hline Female (F) & 9 & $20.02 * *$ & 0.36 & $0.10 * *$ & $10.35^{* *}$ & $2589.78 * *$ & $10.352 * *$ & 0.739 & 0.008 & 0.487 & $0.055 * *$ & $0.518 * *$ & $0.63 * *$ \\
\hline Male (M) & 3 & 3.970 & 0.27 & 0.07 & $21.70^{* *}$ & 4544.40 & $21.709 * *$ & 0.204 & $0.040^{*}$ & 0.109 & $0.070 *$ & 0.024 & $0.98 * *$ \\
\hline Female vs Male (F vs M) & 1 & $62.60 * *$ & $4.16^{* *}$ & 0.00 & 0.008 & 11.07 & 0.008 & 1.523 & 0.000 & 0.759 & 0.014 & $0.451 * *$ & $0.27 * *$ \\
\hline Parents vs. Hybrids (P vs. $\mathrm{H}$ ) & 1 & $91.15^{* *}$ & $27.5 * *$ & $0.34 * *$ & $36.02 * *$ & $13753.62 * *$ & $36.022 * *$ & $12.655^{* *}$ & $0.055 * *$ & $6.795 * *$ & $0.253 * *$ & $6.199^{* *}$ & $0.10^{*}$ \\
\hline Genotype $\mathrm{x}$ Environment & 106 & $11.4 * *$ & $2.26^{* *}$ & $0.04 * *$ & $8.50 * *$ & $1880.67 * *$ & $8.506 * *$ & $0.679 *$ & $0.016^{* *}$ & $0.425 *$ & $0.037 * *$ & $0.388 * *$ & $0.06 * *$ \\
\hline Parents x Environments & 26 & 6.39 & 0.48 & 0.02 & $8.73 * *$ & $1484.68 * *$ & $8.736^{* *}$ & $0.901 *$ & 0.014 & 0.162 & 0.028 & $0.329 * *$ & $0.06 * *$ \\
\hline Females x Environments & 18 & 7.3 & 0.48 & 0.01 & $5.59 * *$ & $991.14 * *$ & $5.596 * *$ & $1.150^{*}$ & 0.011 & 0.182 & 0.024 & $0.248 * *$ & $0.06 * *$ \\
\hline Males x Environments & 6 & 4.05 & 0.35 & 0.04 & $15.86^{* *}$ & $2963.04 * *$ & $15.867 * *$ & $0.399 *$ & 0.022 & 0.096 & 0.032 & $0.604^{* *}$ & $0.07 * *$ \\
\hline (F vs M) x Environment & 2 & 4.34 & 0.92 & 0.07 & $15.60 * *$ & $1491.47 * *$ & $15.603 * *$ & 0.168 & 0.022 & 0.178 & 0.056 & $0.236 * *$ & 0.03 \\
\hline (P vs $\mathrm{H}) \times$ Environment & 2 & 5.35 & $12.4 * *$ & 0.01 & $8.99 *$ & $4480.20 * *$ & $8.999 *$ & 0.192 & 0.009 & $2.028 * *$ & 0.009 & 0.006 & $0.22 * *$ \\
\hline Hybrids x Environment & 78 & $13.2 * *$ & $2.59 * *$ & $0.05^{* *}$ & $8.41 * *$ & $1946.02 * *$ & $8.417 * *$ & 0.618 & $0.017 * *$ & 0.472 & $0.041 * *$ & $0.417^{* *}$ & $0.05 * *$ \\
\hline Pooled Error & 318 & 5.05 & 0.56 & 0.03 & 2.174 & 243.61 & 2.174 & 0.582 & 0.011 & 0.400 & 0.022 & 0.036 & 0.025 \\
\hline
\end{tabular}

(cm), FD: Fruit diameter (cm), FW: Fruit weight (g), SL: Stalk length (cm), CF: Crude fibre content (\%), SLD: Shelf life (days).

Table.5a Per cent standard heterosis (top three crosses) in different environment and across environments for various characters in okra

\begin{tabular}{|c|c|c|c|c|c|c|c|c|}
\hline Character & $\mathrm{E}_{1}$ & $\begin{array}{l}\text { Standard } \\
\text { heterosis }(\%)\end{array}$ & $\mathrm{E}_{2}$ & $\begin{array}{l}\text { Standard } \\
\text { heterosis }(\%)\end{array}$ & $\mathrm{E}_{3}$ & $\begin{array}{l}\text { Standard } \\
\text { heterosis }(\%)\end{array}$ & Pooled & $\begin{array}{l}\text { Standard } \\
\text { heterosis }(\%)\end{array}$ \\
\hline \multirow{3}{*}{$\begin{array}{l}\text { Days to first } \\
\text { flowering }\end{array}$} & VIO $47672 \times$ GJO - 3 & -4.4 & IC $-111493 \times$ GAO -5 & $-11.77 * *$ & VIO 47672 x GJO - 3 & $-5.9 *$ & IC $-111493 \times$ GJO -3 & $-6.49 *$ \\
\hline & IC $-045796 \times$ GAO -5 & -3.5 & VIO $47672 \times$ GAO - 5 & $-11.55^{* *}$ & IC $-111493 \times$ GJO -3 & $-5.27 *$ & VIO $47672 \times$ GJO - 3 & $-6.37 *$ \\
\hline & IC $-111493 \times$ GJO -3 & -2.75 & IC $-111493 \times$ GJO -3 & $-11.44 * *$ & IC $-045796 \times$ GJO -3 & -5.07 & VIO $47672 \times$ GAO -5 & $-6.04 *$ \\
\hline \multirow{3}{*}{$\begin{array}{l}\text { Intermodal } \\
\text { length }(\mathrm{cm})\end{array}$} & AOL - 09-17 x Arka Anamika & $-19.97 * *$ & IC $-052273 \times$ GJO -3 & -12.59 & $\mathrm{JOL}-08-13 \times \mathrm{GJO}-3$ & $-13.53 * *$ & VIO 44244 x GAO - 5 & $-9.93 *$ \\
\hline & EC - 284327 x Arka Anamika & $-17.65 * *$ & VIO 44244 x GAO - 5 & -11.46 & JOL $-08-13 \times$ Varsha Uphar & $-12.52 *$ & JOL $-10-18 \times$ Varsha Uphar & $-9.78 *$ \\
\hline & $\mathrm{EC}-305623 \times \mathrm{GJO}-3$ & $-13.82 *$ & JOL - 10 - 18 x Varsha Uphar & -11.20 & IC - 052273 x Arka Anamika & $-12.23^{*}$ & AOL - $09-17$ x Arka Anamika & $-8.69^{*}$ \\
\hline \multirow{3}{*}{$\begin{array}{l}\text { Number of } \\
\text { branches }\end{array}$} & VIO 47672 x GJO - 3 & $34.80 * *$ & VIO $47672 \times$ GJO - 3 & $19.40 * *$ & VIO 47672 x GJO - 3 & $18.18^{* *}$ & VIO $47672 \times$ GJO - 3 & $24.15^{* * *}$ \\
\hline & IC $-045796 \times$ GAO -5 & $28.63 * *$ & IC $-045796 \times$ GJO -3 & $18.10^{* *}$ & IC $-111493 \times$ GJO -3 & $15.91 * *$ & IC $-111493 \times$ GJO -3 & $19.15^{* *}$ \\
\hline & IC $-111493 \times$ GJO -3 & $24.23^{* *}$ & IC $-111493 \times$ GJO -3 & $17.24 * *$ & IC $-045796 \times$ GJO -3 & $14.09 * *$ & IC $-045796 \times$ GAO -5 & $19.15^{* *}$ \\
\hline \multirow{3}{*}{$\begin{array}{l}\text { Plant height } \\
(\mathrm{cm})\end{array}$} & VIO 47672 x GJO - 3 & 6.06 & VIO 47672 x GJO - 3 & $25.70 * *$ & VIO 47672 x GJO - 3 & $19.96^{* *}$ & VIO 47672 x GJO - 3 & $18.28 * *$ \\
\hline & IC $-045796 \times$ xAO -5 & 4.29 & IC $-045796 \times$ GJO -3 & $23.97 * *$ & IC $-111493 \times$ GJO -3 & $17.73^{* *}$ & IC $-111493 \times$ GJO -3 & $15.50 * *$ \\
\hline & IC $-111493 \times$ GJO -3 & 3.86 & IC $-111493 \times$ GJO - 3 & $22.28 * *$ & IC $-045796 \times$ GJO -3 & $16.51 * *$ & IC $-045796 \times$ GJO -3 & $13.79 * *$ \\
\hline \multirow{3}{*}{$\begin{array}{l}\text { Fruit yield per } \\
\text { plant }(\mathrm{g})\end{array}$} & VIO 47672 x GJO - 3 & $21.17 * *$ & VIO 47672 x GJO - 3 & $36.60 * *$ & VIO 47672 x GJO - 3 & $48.93 * *$ & VIO 47672 x GJO - 3 & $33.30 * *$ \\
\hline & IC $-045796 \times$ GAO -5 & $16.28 * *$ & IC $-045796 \times$ GAO -5 & $27.56^{* *}$ & IC $-045796 \times$ GAO -5 & $45.54 * *$ & IC $-045796 \times$ GAO -5 & $22.83^{* *}$ \\
\hline & IC $-111493 \times$ GJO -3 & $9.52 *$ & IC $-111493 \times$ GJO -3 & $23.45^{* *}$ & IC $-111493 \times$ GJO -3 & $40.05^{* *}$ & IC $-111493 \times$ GJO -3 & $22.27 * *$ \\
\hline \multirow{3}{*}{$\begin{array}{l}\text { Number of } \\
\text { fruits per plant }\end{array}$} & VIO 47672 x GJO - 3 & $13.33^{*}$ & VIO $47672 \times$ GJO - 3 & $19.40^{* *}$ & VIO 47672 x GJO - 3 & $28.15^{* *}$ & VIO 47672 x GJO - 3 & $20.92 * *$ \\
\hline & IC $-045796 \times$ GAO -5 & $9.97 *$ & IC $-045796 \times$ GJO -3 & $18.10^{*}$ & IC $-045796 \times$ GJO -3 & $24.93 * *$ & IC $-045796 \times$ GJO -3 & 15.18 ** \\
\hline & IC $-111493 \times$ GJO -3 & 7.41 & IC $-111493 \times$ GJO -3 & $17.24 *$ & IC $-111493 \times$ GJO -3 & $23.17 * *$ & IC $-111493 \times$ GJO -3 & $15.16^{* *}$ \\
\hline
\end{tabular}


Int.J.Curr.Microbiol.App.Sci (2017) 6(7): 4101-4111

Table.5b Per cent standard heterosis (top three crosses) in different environment and across environments for various characters in okra

\begin{tabular}{|c|c|c|c|c|c|c|c|c|}
\hline Character & $\mathrm{E}_{1}$ & $\begin{array}{l}\text { Standard } \\
\text { heterosis } \\
(\%)\end{array}$ & $\mathrm{E}_{2}$ & $\begin{array}{l}\text { Standard } \\
\text { heterosis } \\
(\%)\end{array}$ & $\mathrm{E}_{3}$ & $\begin{array}{l}\text { Standard } \\
\text { heterosis } \\
(\%)\end{array}$ & Pooled & $\begin{array}{l}\text { Standard } \\
\text { heterosis } \\
(\%)\end{array}$ \\
\hline \multirow{3}{*}{$\begin{array}{l}\text { Fruit length } \\
(\mathrm{cm})\end{array}$} & VIO $47672 \times$ GJO - 3 & $11.17 *$ & VIO $47672 \times$ GJO - 3 & $8.71 *$ & VIO 47672 x GJO - 3 & $21.35^{* *}$ & VIO $47672 \times$ GJO - 3 & $12.72 * *$ \\
\hline & IC $-045796 \times \mathrm{GAO}-5$ & 9.8 & $\mathrm{IC}-045796 \times \mathrm{GAO}-5$ & 7.91 & IC $-045796 \times$ GAO -5 & $19.23 * *$ & $\mathrm{IC}-045796 \times \mathrm{GAO}-5$ & $10.22 * *$ \\
\hline & IC $-111493 \times$ GJO -3 & 8.18 & IC $-111493 \times$ GJO -3 & 6.47 & IC $-111493 \times$ GJO -3 & $17.10 * *$ & IC $-111493 \times$ GJO -3 & $10.07 * *$ \\
\hline \multirow{3}{*}{$\begin{array}{l}\text { Fruit } \\
\text { diameter } \\
(\mathrm{cm})\end{array}$} & VIO $47672 \times$ GJO - 3 & $35.40 * *$ & VIO $47672 \times$ GJO - 3 & $9.76^{*}$ & VIO $47672 \times$ GJO - 3 & $18.44 * *$ & VIO 47672 x GJO - 3 & $20.74 * *$ \\
\hline & IC $-045796 \times$ GAO -5 & $24.90 * *$ & IC $-045796 \times$ GAO -5 & 9.15 & $\mathrm{IC}-045796 \times \mathrm{GAO}-5$ & $12.77 * *$ & IC $-045796 \times$ GAO -5 & $14.67 * *$ \\
\hline & $\mathrm{IC}-111493 \times \mathrm{GJO}-3$ & $24.21 * *$ & IC $-111493 \times$ GJO -3 & 8.54 & IC $-111493 \times$ GJO -3 & $12.06^{* *}$ & $\mathrm{IC}-111493 \times \mathrm{GJO}-3$ & $14.44 * *$ \\
\hline \multirow{3}{*}{$\begin{array}{l}\text { Fruit } \\
\text { weight }(g)\end{array}$} & VIO $47672 \times$ GJO - 3 & $9.44 * *$ & VIO 47672 x GJO - 3 & $11.07 *$ & VIO 47672 x GJO - 3 & $18.14 * *$ & VIO $47672 \times$ GJO - 3 & $12.44 * *$ \\
\hline & IC $-045796 \times$ GAO -5 & $7.84 *$ & IC $-045796 \times$ GAO -5 & 9.71 & IC $-045796 \times$ GAO -5 & $17.58 * *$ & IC $-111493 \times$ GJO -3 & $9.96 * *$ \\
\hline & IC $-111493 \times$ GJO -3 & 5.34 & IC $-111493 \times$ GJO -3 & 8.68 & IC $-111493 \times$ GJO -3 & $14.56^{*}$ & $\mathrm{IC}-045796 \times \mathrm{GAO}-5$ & $8.83^{* *}$ \\
\hline \multirow[t]{3}{*}{$\begin{array}{l}\text { Stalk } \\
\text { length }(\mathrm{cm})\end{array}$} & $\begin{array}{l}\text { VIO } 44244 \text { x Arka } \\
\text { Anamika }\end{array}$ & -3.13 & EC - 284327 x Varsha Uphar & -8.68 & $\begin{array}{l}\text { VIO } 44244 \text { x Arka } \\
\text { Anamika }\end{array}$ & - & $\begin{array}{l}\text { VIO } 44244 \text { x Arka } \\
\text { Anamika }\end{array}$ & $-8.80 *$ \\
\hline & $\begin{array}{l}\text { IC - } 111493 \times \text { Varsha } \\
\text { Uphar }\end{array}$ & -3.13 & VIO 44244 x Arka Anamika & -6.61 & IC - 111493 x GJO - 3 & - & IC - 111493 x GJO - 3 & $-8.66^{*}$ \\
\hline & IC -111493 x GJO - 3 & -2.68 & IC $-111493 \times$ x GJO - 3 & -6.61 & $\mathrm{EC}-284327 \times \mathrm{GJO}-3$ & $-12.80^{* *}$ & $\begin{array}{l}\text { IC - } 111493 \text { x Varsha } \\
\text { Uphar }\end{array}$ & -4.89 \\
\hline \multirow[t]{3}{*}{$\begin{array}{l}\text { Crude fiber } \\
\text { content }(\%)\end{array}$} & $\begin{array}{l}\text { VIO } 47672 \text { x Arka } \\
\text { Anamika }\end{array}$ & $-9.77 * *$ & VIO 47672 x Varsha Uphar & $-13.25 * *$ & $\begin{array}{l}\text { IC - } 111493 \times \text { V Varsha } \\
\text { Uphar }\end{array}$ & $-\overline{18.81 * *}$ & VIO 44244 x GJO - 3 & $-9.48 * *$ \\
\hline & VIO 44244 x GJO - 3 & $-9.56 * *$ & IC - 052273 x Varsha Uphar & - & VIO 44244 x GJO - 3 & $-18.14 * *$ & $\begin{array}{l}\text { IC - } 045796 \times \text { Varsha } \\
\text { Uphar }\end{array}$ & $-6.34 * *$ \\
\hline & IC $-111493 \times$ GJO - 3 & $-7.90 *$ & $\begin{array}{l}\text { AOL - } 09-17 \text { x Arka } \\
\text { Anamika }\end{array}$ & -8.09 & $\begin{array}{l}\text { IC }-111493 \text { x Arka } \\
\text { Anamika }\end{array}$ & $-\overline{13.72 * *}$ & $\begin{array}{l}\text { IC }-045796 \times \text { Arka } \\
\text { Anamika }\end{array}$ & $-6.13 * *$ \\
\hline \multirow[t]{3}{*}{$\begin{array}{l}\text { Shelf life } \\
\text { (days) }\end{array}$} & $\begin{array}{l}\text { IC - } 111493 \times \text { V Varsha } \\
\text { Uphar }\end{array}$ & $10.80^{* *}$ & $\mathrm{JOL}-08-13 \times \mathrm{GJO}-3$ & $15.95 * *$ & $\mathrm{JOL}-08-13 \times \mathrm{GJO}-3$ & 5.75 & $\mathrm{AOL}-09-17 \times \mathrm{GJO}-3$ & $19.88 * *$ \\
\hline & $\mathrm{JOL}-08-13 \times \mathrm{GJO}-3$ & $10.51 * *$ & IC - 111493 x Varsha Uphar & $9.62 * *$ & $\begin{array}{l}\text { VIO } 47672 \text { x Arka } \\
\text { Anamika }\end{array}$ & 4.83 & $\mathrm{JOL}-08-13 \times \mathrm{GJO}-3$ & $10.58 * *$ \\
\hline & $\begin{array}{l}\text { VIO } 47672 \text { x Arka } \\
\text { Anamika }\end{array}$ & $6.82 * *$ & VIO 47672 x Arka Anamika & $7.59 * *$ & $\mathrm{EC}-284327 \times \mathrm{GAO}-5$ & 1.61 & $\begin{array}{l}\text { IC - } 111493 \text { x Varsha } \\
\text { Uphar }\end{array}$ & $6.43 * *$ \\
\hline
\end{tabular}


Int.J.Curr.Microbiol.App.Sci (2017) 6(7): 4101-4111

Table.6 Fruit stalk colour of okra parents and $\mathrm{F}_{1}$ hybrids

\begin{tabular}{|c|c|c|c|c|c|c|c|c|}
\hline Parents & & $\begin{array}{l}\text { Stalk } \\
\text { colour }\end{array}$ & Hybrids & $\begin{array}{l}\text { Stalk } \\
\text { colour }\end{array}$ & Hybrids & $\begin{array}{l}\text { Stalk } \\
\text { colour }\end{array}$ & Hybrids & $\begin{array}{l}\text { Stalk } \\
\text { colour }\end{array}$ \\
\hline \multirow[t]{10}{*}{ Lines } & VIO 44244 & $\begin{array}{l}\text { Light } \\
\text { green }\end{array}$ & VIO 44244 x GAO - 5 & $\begin{array}{l}\text { Light } \\
\text { green }\end{array}$ & $\begin{array}{l}\text { EC - } 284327 \text { x Arka } \\
\text { Anamika }\end{array}$ & $\begin{array}{l}\text { Light } \\
\text { green }\end{array}$ & $\mathrm{AOL}-09-17 \times \mathrm{GAO}-5$ & $\begin{array}{l}\text { Light } \\
\text { green }\end{array}$ \\
\hline & IC -111493 & $\begin{array}{l}\text { Medium } \\
\text { green }\end{array}$ & VIO 44244 x GJO - 3 & $\begin{array}{l}\text { Light } \\
\text { green }\end{array}$ & $\begin{array}{l}\text { EC - } 284327 \text { x Varsha } \\
\text { Uphar }\end{array}$ & $\begin{array}{l}\text { Light } \\
\text { green }\end{array}$ & $\mathrm{AOL}-09-17 \times \mathrm{GJO}-3$ & $\begin{array}{l}\text { Medium } \\
\text { green }\end{array}$ \\
\hline & $\begin{array}{l}\mathrm{JOL}-08- \\
13\end{array}$ & $\begin{array}{l}\text { Light } \\
\text { green }\end{array}$ & $\begin{array}{l}\text { VIO } 44244 \text { x Arka } \\
\text { Anamika }\end{array}$ & $\begin{array}{l}\text { Light } \\
\text { green }\end{array}$ & $\mathrm{IC}-045796 \times \mathrm{GAO}-5$ & $\begin{array}{l}\text { Light } \\
\text { green }\end{array}$ & $\begin{array}{l}\text { AOL }-09-17 \text { x Arka } \\
\text { Anamika }\end{array}$ & $\begin{array}{l}\text { Light } \\
\text { green }\end{array}$ \\
\hline & $\begin{array}{l}\mathrm{EC}- \\
284327\end{array}$ & $\begin{array}{l}\text { Light } \\
\text { green }\end{array}$ & $\begin{array}{l}\text { VIO } 44244 \text { x Varsha } \\
\text { Uphar }\end{array}$ & $\begin{array}{l}\text { Light } \\
\text { green }\end{array}$ & IC -045796 x GJO - 3 & $\begin{array}{l}\text { Dark } \\
\text { green }\end{array}$ & $\begin{array}{l}\text { AOL }-09-17 \times \text { Varsha } \\
\text { Uphar }\end{array}$ & $\begin{array}{l}\text { Light } \\
\text { green }\end{array}$ \\
\hline & IC -045796 & $\begin{array}{l}\text { Medium } \\
\text { green }\end{array}$ & $\mathrm{IC}-111493 \times \mathrm{GAO}-5$ & $\begin{array}{l}\text { Light } \\
\text { green }\end{array}$ & $\begin{array}{l}\text { IC - } 045796 \text { x Arka } \\
\text { Anamika }\end{array}$ & $\begin{array}{l}\text { Light } \\
\text { green }\end{array}$ & VIO 47672 x GAO - 5 & $\begin{array}{l}\text { Medium } \\
\text { green }\end{array}$ \\
\hline & $\mathrm{IC}-052273$ & $\begin{array}{l}\text { Light } \\
\text { green }\end{array}$ & IC - 111493 x GJO - 3 & $\begin{array}{l}\text { Medium } \\
\text { green }\end{array}$ & IC - 045796 x Varsha Uphar & $\begin{array}{l}\text { Light } \\
\text { green }\end{array}$ & VIO 47672 x GJO - 3 & $\begin{array}{l}\text { Dark } \\
\text { green }\end{array}$ \\
\hline & $\begin{array}{l}\mathrm{JOL}-10- \\
18\end{array}$ & $\begin{array}{l}\text { Light } \\
\text { green }\end{array}$ & $\begin{array}{l}\text { IC - } 111493 \text { x Arka } \\
\text { Anamika }\end{array}$ & $\begin{array}{l}\text { Medium } \\
\text { green }\end{array}$ & $\mathrm{IC}-052273 \times \mathrm{GAO}-5$ & $\begin{array}{l}\text { Dark } \\
\text { green }\end{array}$ & VIO 47672 x Arka Anamika & $\begin{array}{l}\text { Light } \\
\text { green }\end{array}$ \\
\hline & $\begin{array}{l}\mathrm{AOL}-09- \\
17\end{array}$ & $\begin{array}{l}\text { Light } \\
\text { green }\end{array}$ & $\begin{array}{l}\text { IC }-111493 \times \text { Varsha } \\
\text { Uphar }\end{array}$ & $\begin{array}{l}\text { Light } \\
\text { green }\end{array}$ & $\mathrm{IC}-052273 \times \mathrm{GJO}-3$ & $\begin{array}{l}\text { Light } \\
\text { green }\end{array}$ & VIO 47672 x Varsha Uphar & $\begin{array}{l}\text { Light } \\
\text { green }\end{array}$ \\
\hline & VIO 47672 & $\begin{array}{l}\text { Medium } \\
\text { green }\end{array}$ & $\mathrm{JOL}-08-13 \times \mathrm{GAO}-5$ & $\begin{array}{l}\text { Light } \\
\text { green }\end{array}$ & $\begin{array}{l}\text { IC - } 052273 \text { x Arka } \\
\text { Anamika }\end{array}$ & $\begin{array}{l}\text { Light } \\
\text { green }\end{array}$ & $\mathrm{EC}-305623 \times \mathrm{GAO}-5$ & $\begin{array}{l}\text { Dark } \\
\text { green }\end{array}$ \\
\hline & $\begin{array}{l}\mathrm{EC}- \\
305623\end{array}$ & $\begin{array}{l}\text { Light } \\
\text { green }\end{array}$ & $\mathrm{JOL}-08-13 \times \mathrm{GJO}-3$ & $\begin{array}{l}\text { Light } \\
\text { green }\end{array}$ & IC - 052273 x Varsha Uphar & $\begin{array}{l}\text { Dark } \\
\text { green }\end{array}$ & $\mathrm{EC}-305623$ x GJO - 3 & $\begin{array}{l}\text { Light } \\
\text { green }\end{array}$ \\
\hline \multirow[t]{4}{*}{ Testers } & $\mathrm{GAO}-5$ & $\begin{array}{l}\text { Dark } \\
\text { green }\end{array}$ & $\begin{array}{l}\text { JOL }-08-13 \times \text { Arka } \\
\text { Anamika }\end{array}$ & $\begin{array}{l}\text { Dark } \\
\text { green }\end{array}$ & $\mathrm{JOL}-10-18 \times \mathrm{GAO}-5$ & $\begin{array}{l}\text { Light } \\
\text { green }\end{array}$ & $\begin{array}{l}\mathrm{EC}-305623 \times \text { x Arka } \\
\text { Anamika }\end{array}$ & $\begin{array}{l}\text { Light } \\
\text { green }\end{array}$ \\
\hline & $\mathrm{GJO}-3$ & $\begin{array}{l}\text { Dark } \\
\text { green }\end{array}$ & $\begin{array}{l}\text { JOL }-08-13 \times \text { Varsha } \\
\text { Uphar }\end{array}$ & $\begin{array}{l}\text { Light } \\
\text { green }\end{array}$ & $\mathrm{JOL}-10-18 \times \mathrm{GJO}-3$ & $\begin{array}{l}\text { Medium } \\
\text { green }\end{array}$ & $\begin{array}{l}\text { EC - } 305623 \times \text { Varsha } \\
\text { Uphar }\end{array}$ & $\begin{array}{l}\text { Light } \\
\text { green }\end{array}$ \\
\hline & $\begin{array}{l}\text { Arka } \\
\text { Anamika }\end{array}$ & $\begin{array}{l}\text { Dark } \\
\text { green }\end{array}$ & $\mathrm{EC}-284327 \times \mathrm{GAO}-5$ & $\begin{array}{l}\text { Light } \\
\text { green }\end{array}$ & $\begin{array}{l}\text { JOL }-10-18 \times \text { Arka } \\
\text { Anamika }\end{array}$ & $\begin{array}{l}\text { Light } \\
\text { green }\end{array}$ & CHECK (Sonakshi) & $\begin{array}{l}\text { Dark } \\
\text { green }\end{array}$ \\
\hline & $\begin{array}{l}\text { Varsha } \\
\text { Uphar }\end{array}$ & $\begin{array}{l}\text { Dark } \\
\text { green }\end{array}$ & $\mathrm{EC}-284327 \times \mathrm{GJO}-3$ & $\begin{array}{l}\text { Medium } \\
\text { green }\end{array}$ & $\begin{array}{l}\text { JOL }-10-18 \times \text { Varsha } \\
\text { Uphar }\end{array}$ & $\begin{array}{l}\text { Light } \\
\text { green }\end{array}$ & & \\
\hline
\end{tabular}


VIO $47672 \times$ GJO - $3(12.72 \%, 20.74 \%$ and $12.44 \%)$, IC $-045796 \times$ GAO - 5 (10.22\%, $14.67 \%$ and $8.83 \%)$ and IC $-111493 \times$ GJO $-3(10.07 \%, 14.44 \%$ and $9.96 \%)$ were the top three hybrids for fruit length, fruit diameter and fruit weight, respectively. In the present investigation, the maximum standard heterosis for fruit yield per plant recorded by cross combination VIO 47672 x GJO - 3 $(33.33 \%)$ followed by IC - $045796 \times$ GAO $5(22.83 \%)$ and IC -111493 x GJO - 3 $(22.27 \%)$.

It is interesting to note that top ranking crosses based on per se performance and standard heterosis was the same. The high fruit yield recorded in the hybrid from the VIO 47672 x GJO - 3 cross can be directly attributed to earlier flowering (-6.37\%), increased number of branches per plant (24.15\%), number of fruits per plant $(20.92 \%)$, fruit length $(12.72 \%)$, fruit diameter $(20.74 \%)$ and fruit weight $(12.44 \%)$ because they are very closely related yield components. Heterosis for growth parameters is an indication of heterosis for yield as growth and yield parameters are strongly associated. Heterosis is thought to result from the combined action and interaction of allelic and non-allelic factors and is usually closely and positively correlated with heterozygosity (Falconer, 1952). Similar observations were reported earlier in okra by many researchers like Kishor et al., (2013), Obiadalla et al., (2013), Kumar et al., (2014), Akotkar et al., (2014), Badiger et al., (2014) and Katagi et al., (2015).

In okra, the fruit length, fruit diameter and fruit weight are the important yield components. Such high heterotic response would be useful for obtaining higher fruit yield. Both the parents and hybrids exhibited wider variation for fruit weight. Such an increase in fruit weight can be attributed to the conglomeration of favorable genes in the hybrids (Das et al., 2013).

\section{Estimation of heterosis for quality parameters}

Very few researchers have studied the heterosis for quality traits in okra. Okra fruits with shorter stalk length and lower crude fibers are considered as good in quality. Cross of VIO $44244 \times$ Arka Anamika (-8.80\%) and IC -111493 x GJO - $3(-8.66 \%)$ exhibited heterosis in desired direction for stalk length. Cross combination, VIO 44244 x GJO - 3 ($9.48 \%$ ) resulted in the lowest significant and negative heterosis for this trait in pooled analysis for crude fiber content (Table 5b).

In present study, negative and significant heterosis for stalk length and crude fiber observed which is in confirmation with the findings of Shobha and Marriappan (2007), Indurani et al., (2003), Kumar et al., (2014) and Flemine Xavier et al., (2016). AOL - 09 -17 x GJO - 3, JOL - $08-13$ x GJO - 3 and IC $-111493 \times$ Varsha Uphar are the top three crosses which resulted in significant and positive heterosis over standard check for shelf life. The present findings are in agreement with results reported by Patwary et al., (2013), Narasimhamurthy and Gowda (2013) and Garg et al., (2013) in tomato.

\section{Visual observation of fruit stalk colour}

The colour of the fruit and fruit stalk (Table 6) are important quality parameters in okra. Consumers prefer mostly dark green fruit. In the present investigation, three colour shades were observed in fruits of okra viz., Dark Green, Medium Green and Light Green. Among all the parents, lines, IC - 111493, IC - 045796 and VIO 47672 had medium green stalk colour and rest of all had light green stalk colour. Dark green stalk colour is a desirable trait as per the quality point of view. Hybrids viz; IC - 045796 x GJO - 3, IC 052273 x GAO - 5, IC - 052273 x Varsha Uphar, VIO 47672 x GJO - 3 and EC - 
305623 x GAO - 5 exhibited dark green stalk colour. Colour of fruit was also reported by Alake and Ariyo (2012) and Solankey et al., (2013). In the present study, the significance of the heterotic performance was highly affected by the genetic background of parental genotypes. The high heterosis among these hybrids for most of the characteristics studied indicates that the considerable potential exist in parents for developing hybrids. The outstanding hybrids $\left(\mathrm{F}_{1}\right) \mathrm{VIO}$ 47672 x GJO - 3, IC - 045796 x GAO - 5 and IC - $111493 \times$ xJO - 3 were good for fruit yield during all the seasons. These hybrids can be evaluated further to satisfy the local demand.

\section{Acknowledgement}

The author is thankful to the Department of Science \& Technology, Govt. of India for felicitating INSPIRE fellowship for financial support.

\section{References}

Akotkar P. K., De D. K. and Dubey U. 2014. Genetic studies on fruit yield and yield attributes of okra (Abelmoschus esculentus (L.) Moench). Electron. J. Plant Breed. 5(1): 38 - 44.

Alake C. O. and Ariyo O. J. 2012. Comparative analysis of genotype $\mathrm{x}$ environment interaction in West African okra (Abelmoschus esculentus (L.) Moench). Journal of Agricultural Science 4(4): 135 -150 .

Anonymous. 2011. Series of Crop Specific Biology Documents, Biology of Abelmoschus esculentus L. (Okra). Department of Biotechnology, Ministry of Science and Technology and Ministry of Environment and Forests, Govt. of India. pp: $3-4$.

Anonymous. 2015. Indian Horticulture Database, National horticulture Board. www. nhb.gov.in.
Badiger M., Pitchaimuthu M., Parvati, B. and Sachin U. 2014. Heterosis studies in conventional and genetic male sterility based hybrids in okra (Abelmoschus esculentus). Green Farming International Journal 5(4):524 - 528.

Charrier A. 1984. Genetic resources of the genus Abelmoschus Med. (Okra). IBPGR, Italy, pp. 61.

Das S., Chattopadhyay A., Dutta S., Chattopadhyay S., and Hazra P. 2013. Breeding okra for higher productivity and yellow vein mosaic tolerance. International Journal of Vegetable Science 19(1): $58-77$.

Falconer D. S. 1952. Selection for large and small size in mice. J. Genet. 51: 470 501.

FAOSTAT. 2013. Production- Crops (2013) data. Food and Agriculture Organization of the United Nations. http://www.fao.org/faostat

Flemine Xavier, Raj Kumar, Behera T. K. and Yadav, R. K. 2016. Studies on heterosis for qualitative traits in okra. Indian J. Hortic. 73(4): 532 - 537.

Garg N., Cheema D. S. and Neena Chawla. 2013. Manifestation of heterosis for fruit yield, quality and shelf-life in tomato (Solanum lycopersicum L.) hybrids incorporating rin, nor or alc alleles in main season and late season of north Indian plains. Veg. Sci. 40(1): $28-33$.

Indurani C., Veeraragavathatham D. and Auxcilia J. 2003. Studies on the development of $\mathrm{F}_{1}$ hybrids in okra (Abelmoschus esculentus (L.) Moench) with high yield and resistance to yellow vein mosaic virus. South Indian Hortic. 51(1-6): $219-226$.

Katagi A., Tirakannanvar S. and Jagadeesha R. C. 2015. Combining ability through diallel analysis in okra (Abelmoschus esculentus (L.) Moench). Green Farming International Journal 6(1): 26 - 29.

Kishor D. S., Arya K., Duggi Shrishail, Magadum S., Raghavendra N. R., 
Venkateshwaralu Challa and Reddy P. S. 2013. Studies on heterosis for yield and yield contributing traits in okra (Abelmoschus esculentus (L.) Moench). Mol. Breed. 4(35): 277 - 284.

Kumar Rahul R. K., Yadav Rakesh Bhardwaj, Baranwal V. K. and Chaudhary H. 2016. Studies on genetic variability of nutritional traits among YVMV tolerant okra germplasm. Indian J. Hortic. 73(2): 202-207.

Kumar S., Singh A. K., Das R., Datta S. and Arya K. 2014. Combining ability and its relationship with gene action in okra [Abelmoschus esculentus (L.) Meonch]. J. Crop Weed 10(1): 82 - 92.

Narasimhamurthy Y. K. and Gowda P. H. R. 2013. Line $\mathrm{x}$ tester analysis in tomato (Solanum lycopersicum L.): Identification of superior parents for fruit quality and yield attributing traits. International Journal of Plant Breeding 7(1): 50 - 54.

Obiadalla Ali, Eldekashi M. H. and Helaly A. A. 2013. Combining ability and heterosis studies for yield and its components in some cultivars of okra. Am. Eurasian J. Agric. Environ. Sci. 13(2): 162 - 167.

Panse V. G. and Sukhatme P. V. 1967. Statistical Methods for Agricultural Workers, Indian Council of Agricultural Research, New Delhi, India, pp. 152 161.

Patel R. K. 2014. Manifestation of Heterosis in Okra (Abelmoschus esculentus (L.) Moench). Trends in Biosciences 7(5): 322-328.

Patwary M. M. A., Rahman M. M., Ahmad S., Khaleque M. A. and Barua H. 2013.
Study of heterosis in heat tolerant tomato (Solanum lycopersicum) during summer. Bangladesh J. Agric. Res. 38(3): 531 544.

Reddy T. M., Babu K. H., Ganesh M., Hameedunnisa Begam, Dilipbabu J. and Reddy R. S. K. 2012. Gene action and combining ability of yield and its components for late Kharif season in okra (Abelmoschus esculentus (L.) Meonch). Chil. J. Agr. Res. 73(1): 9 - 16.

Sharma J. P. and Singh A. K. 2012. Line x Tester analysis for combining ability in okra (Abelmoschus esculentus (L.) Moench). Veg. Sci. 39(2): 132 - 135.

Shobha K. and Marriappan S. 2007. Heterosis studies in okra (Abelmoschus esculentus (L.) Moench) for some important biometrical traits. Acta Hortic. 752: 437 438.

Singh R. K. and Chaudhary B. D. 1977. Biometrical Methods in Quantitative Genetic Analysis. Kalyani Publishers, New Delhi, India.

Solankey S. S., Singh A. K. and Singh R. K. 2013. Genetic expression of heterosis for yield and quality traits during different growing seasons in okra (Abelmoschus esculentus (L.) Moench). Indian J. Agr. Sci. 83(8): $815-819$.

Thimmaiah S. R. 1999. Standard methods of biochemical analysis. Kalyani Publishers, New Delhi. pp. $64-65$.

Vijayaraghavan C. and Warrier V. A. 1946. Evaluation of high yielding hybrids in bhendi (Hibischus esculentus). Proceedings of $33^{\text {rd }}$ Indian Science Congress 33 (33).

\section{How to cite this article:}

Sanket J. More, K.N. Chaudhari, G.B. Vaidya and Chawla, S.L. 2017. Estimation of Hybrid Vigour for Fruit Yield and Quality Traits of Okra [Abelmoschus esculentus (L.) Moench] Through Line x Tester Analysis Carried Over Environments. Int.J.Curr.Microbiol.App.Sci. 6(7): 4101-4111. doi: https://doi.org/10.20546/ijcmas.2017.607.425 